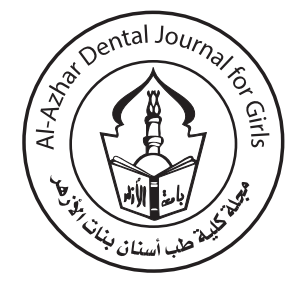

\title{
A Comparative Study for the Effectiveness of Chemiluminescence and Toluidine Blue in the Early Detection of Oral Cancers
}

\section{Mosaad A. Khalifah ${ }^{1^{*}}$}

Codex : 15/21.01

azhardentj@azhar.edu.eg

http://adjg.journals.ekb.eg

DOI: $10.21608 /$ adjg.2021.46936.1307

Oral Medicine \& Surgical Sciences (Oral Medicine, Oral \& Maxillofacial Surgery, Oral Pathology, Oral Biology)

\section{KEYWORDS}

Dysplasia, Toluidine blue,

Vizilite, Chemiluminescence

\begin{abstract}
Purpose: This study aimed at comparing the efficacy of chemiluminescence and toluidine blue in detecting oral dysplasias and malignancies in at an early stage. Material and methods: A total of 26 patients who had potentially malignant lesions were included in the current study. All lesions were examined using Vizilite ${ }^{\text {TM }}$ chemiluminescence followed by painting them with toluidine blue biostain. Then, all lesions were biopsied to be the gold standard for assessing both the chemiluminescence and the toluidine blue. Results: The current study showed low sensitivity, but high specificity and positive predictive value of TB $(57.15 \%, 60 \%$, and $85.71 \%$ respectively). On contrary, were the results of Vizilite $(85.71 \%, 25 \%$, and $57.14 \%$ on order). Conclusion: Although biopsy is the gold standard to detect dysplasia, toluidine blue and Vizilite were valuable non-invasive and rapid adjunct techniques that aided in the clinical detection and screening of dysplasia. They might have a special value in the screening for the existence of new lesions in patients with previous oral malignancies.
\end{abstract}

\section{INTRODUCTION}

Squamous cell carcinoma (SCC) in the oral region is the sixths most common malignancy worldwide ${ }^{(1)}$. It has a direct medical, social, and financial burden due to the high mortality rate and the marked morbidity manifested in the facial disfigurement, impaired speech and malnutrition as a sequela of the oral malignancies itself or its management. The paramount importance of early diagnosis of oral cancers and precancerous lesions cannot be overemphasized, since early detection and treatment of oral cancers raises the 5 year survival rates up to $80 \%$ and improves the quality of life ${ }^{(2,3)}$. Precancerous lesions are associated

1. Lecturer of Oral and Maxillofacial Surgery, Oral and Maxillofacial Surgery Department, Faculty of Dentistry, Kafr Elsheikh University.

* Corresponding author email: mosaad_khalifa@den.kfs.edu.eg 
with a significantly increased risk of cancer development, so as that a frank cancer was revealed in $16 \%$ of cases diagnosed as leukoplakia. Although biopsy is the gold standard to determine if the suspected tissue is dysplastic or not, it is invasive and might cause tumor seeding. For that reason, various methods were used as an adjunct for the clinical examination for the early detection of cancerous lesions including toluidine blue (TB), and Vizilite ${ }^{(4,5)}$.

Toluidine blue (tolonium chloride) is an acidophilic metachromatic dye has a selective ability to stain acidic tissues and thus nucleic acid. Toluidine Blue can relatively detect the differences between normal and dysplastic and malignant cells and tissue, due to its affinity to dysplastic tissues due to allelic loss, loss of heterozygosity and or increased membrane permeability of these cells, which allows the stain to pass into them. The increased nuclearcytoplasmic ratio of dysplastic cells causes an enhancement of the intracellular binding capacity of $\mathrm{TB}^{(6,7)}$.

Chemiluminescence is another diagnostic adjunct for the detection of the dysplasias. Although bioluminescence dated as far back as 1500 BC, chemiluminescence was a relatively modern discovery commenced at the year 1669 in Germany by the physician Hening Brand; and moreover, it was not before 1888 to introduce the term "chemiluminescnce" by Eilhardt Weidemann ${ }^{(8)}$. Vizilite is a recently introduced diagnostic tool devised for the simple and early diagnosis of oral cancer and is based on the principle of chemiluminescence. It has been shown to be an easy, quick, safe and non-invasive technique that is capable of detecting even the early, asymptomatic precancerous and cancerous lesions in the oral cavity ${ }^{(9)}$. Its mechanism of action is that the blue white light is absorbed by the normal mucosal cells while being reflected by cells with abnormal nuclei. The normal mucosa appears blue, whereas abnormal mucosa reflects the light and appears brighter (aceto-white), sharper, and more with distinct margins ${ }^{(10)}$.
Although a plethora of the techniques (including TB and Vizilite) and devices employed for the early detection of oral cancers, its effectiveness is still questionable. Therefore, the current study aimed to evaluate the effectiveness of TB and Vizilite as screening and detection techniques of oral cancers.

\section{MATERIAL AND METHODS}

A total of 26 patients ( 21 males and five females (statistically significant difference at $\alpha=0.01 ; \mathrm{P}=$ 0.0024; Fisher's exact test)) were included in the current study according to the following criteria. Inclusion criteria involved patients clinically diagnosed as having potentially malignant lesion such as leukoplakia, Erythroplakia, Oral Submucous fibrosis or Oral lichen planus. However, exclusion criteria involved patients already diagnosed with frank oral cancer and patients with any systemic disease, mentally unstable, and or unable to cooperate.

All patients were asked to rinse their mouth with water for about 30 seconds to remove debris followed by rinsing the mouth with the Vizilite ${ }^{\mathrm{TM}}$ rinse (1\% acetic acid solution) for one minute. The room light was dimmed to minimize the ambient light. The Vizilite TM capsule was activated by sharp bending, and assembled within the Vizilite retractor. The oral cavity was re-examined using the illumination from the Vizilite assembly (Fig. 1). The presence of a characteristic "acetowhite" area was considered to be a "positive" result. The absence of such findings was considered a "negative" result.

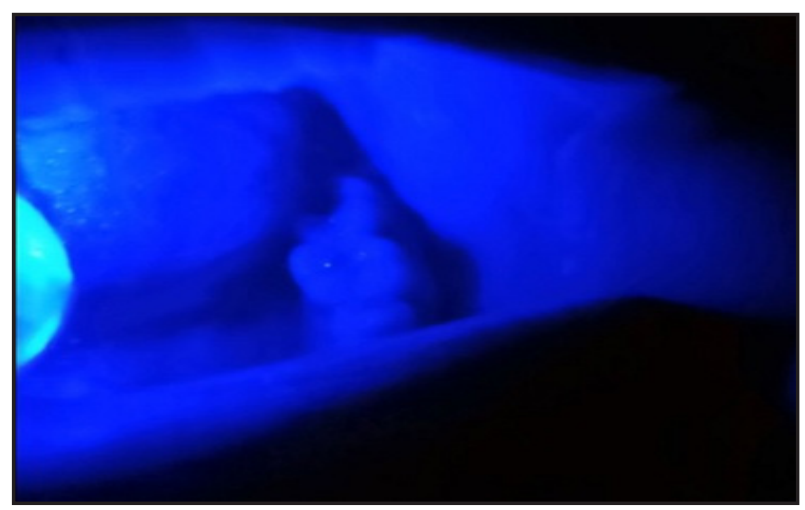

Figure (1) Examination using Vizilite technique. 
Then, all patients were asked to rinse their mouth with for 30 seconds followed by mopping the mucosa with $1 \%$ acetic acid. A cotton applicator tip was soaked with toluidine blue (Toluidine Blue, El-Gomhouria Co. for Trading Drugs, Chemicals \& Medical Supplies, Cairo, Egypt) was used to apply the dye over the same lesion area for 30 seconds. $1 \%$ acetic acid on a cotton applicator tip was used again, to remove excess stain from the lesion (Fig. 2 ). Visual examination was repeated under standard incandescent light to identify if TB stain was retained which means a positive result or it was removed and hence a negative result was recorded.

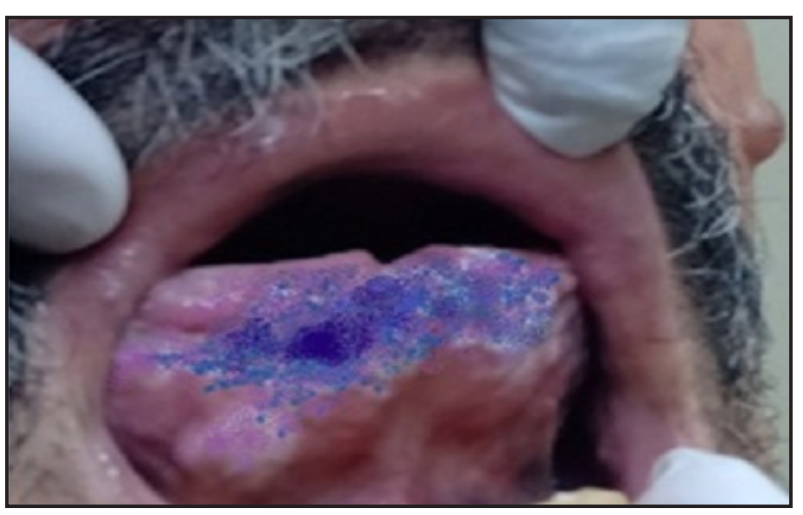

Figure (2) Examination using toluidine blue.

All lesions were then biopsied using scalpel technique and a histopathological examination was performed. Positive result was recorded if dysplasia was revealed and then was subdivided into mild dysplasia, moderate dysplasia, severe dysplasia, carcinoma in situ, and invasive carcinoma). Negative result was considered if no dysplasia was revealed as in lesions such as acanthosis, inflammatory lesions, etc. Histopathological examination was considered the gold standard examination method against which the other two methods were compared.

This study complied with the Declaration of Helsinki (revised in 1975), and with CONSORT (Consolidated Standards of Reporting Trials) principles and the regional ethical review board. All patients provided informed consent. Statistics were performed with SPSS software (version 19, IBM Co, USA). Sensitivity, specificity, and positive predictive values (PPV) for TB and Vizilite TM technique were calculated using the UK Centre for Evidence Based Medicine's online calculator for diagnostic tests ${ }^{(11)}$.

\section{RESULTS}

As table 1 shows, leukoplakia was the most common clinical finding (57.69\%). On comparing the results of both TB and Vizilite with the histopathologic findings, it could be noted that the sensitivity, specificity, and PPV for TB were $57.15 \%$, $60 \%$, and $85.71 \%$ on order and for Vizilite were $85.71 \%, 25 \%$, and 57.14 respectively. The difference between both techniques regarding sensitivity, specificity, and PPV was statistically significant $(\mathrm{P}=0.0231),(\mathrm{P}=0.0001)$, and $(\mathrm{P}=0.0231)$ respectively; all at $\alpha$ level $=5 \%$ using Fisher's exact test.

Table (1) Positive and negative values for TB and Vizilite tests in relation to histopathologic findings

\begin{tabular}{|c|c|c|c|c|c|c|}
\hline \multirow{2}{*}{$\begin{array}{c}\text { Clinical diagnosis followed } \\
\text { by number of cases }\end{array}$} & \multicolumn{2}{|c|}{ TB } & \multicolumn{2}{c|}{ Vizilite } & \multicolumn{2}{c|}{ Histopathologic diagnosis } \\
\cline { 2 - 7 } & Positive & Negative & Positive & Negative & Dysplasia & No dysplasia \\
\hline Leukoplakia 15 & 9 & 6 & 14 & 1 & 13 & 2 \\
\hline Oral lichen planus 4 & 2 & 2 & 3 & 1 & 1 & 2 \\
\hline Frictional keratosis 3 & 0 & 3 & 3 & 0 & 2 & 0 \\
\hline Erythroplakia 2 & 2 & 0 & 0 & 0 & 0 & 1 \\
\hline Actinic keratosis 1 & 1 & 0 & 1 & 1 & 0 & 1 \\
\hline Lichenoid reaction 1 & 0 & 12 & 21 & 5 & 17 & 9 \\
\hline Total & 14 & & 0 & 2 & 0 \\
\hline
\end{tabular}




\section{DISCUSSION}

Oral cancers are very common diseases that could cause considerable mortalities and morbidities, and therefore, early detection and treatment of cancer and precancerous lesions raise the survival rates improves the quality of life ${ }^{(1-3)}$. One of the methods of the early detection of oral cancers is TB which depends for its action on the higher nuclear content and the increased membrane permeability of the dysplastic cells ${ }^{(7)}$. Although TB has been used for a long time as a substitute for frozen sections ${ }^{(2)}$, it provides informations only about surface changes as it stains two to ten layers of cells. This might result in false negative results as in cases of homogenous leukoplakia due to the thick layer of keratin ${ }^{(12)}$.

Vizilite is a more recent tool uses the principle of chemiluminescence by the reaction of acetyl salicylic acid and hydrogen peroxide to release energy (chemiluminescent light), The acetic acid solution used before the activation of the capsule disrupts the glycoprotein barrier on the surface of the epithelium allowing penetration of the light and causes cytoplasmic dehydration which changes its light refractile properties so that when chemiluminescent light is applied, it is absorbed by normal mucosal cells which appears blue where the light is reflected by the dysplastic cells to appear as white (acetowhite), Vizilite detects the metabolic and structural changes in the mucosa which have different absorbance and reflectance properties from those of the normal tissues ${ }^{(9,13,14)}$.

Although various methods were used for the early detection of cancerous lesions including TB and Vizilite, its effectiveness is still questionable. Therefore, the current study aimed to evaluate the effectiveness of TB and Vizilite in the early detection of oral cancers.

In the current study, TB had low sensitivity but higher specificity with very high PPV reaches $85.71 \%$. That was in accordance with various studies, but on contrary with Bagga et al (7, 15-19). On the other hand, high sensitivity and low specificity and PPV were recorded in the current study for Vizilite; in agreement with many studies, but in disagreement with mehrotra et $\mathrm{al}^{(6,8,17,19)}$. That means that while using TB could lead to missing some lesions, depending on Vizilite would lead to many false positives and hence, many unnecessary biopsies. However, biopsy weather using the punch or the scalpel is still the gold standard technique to detect dysplasias. However, TB and Vizilite are reliable non-invasive techniques for early detection and screening of precancerous lesions.

\section{CONCLUSION}

Oral malignancies lead to considerable health and financial sequelae, so that early detection of dysplasia is mandatory for early treatment and to decrease these sequalae. Despite the various techniques applied to early detect dysplasia, its effect is equivocal. The current study showed low sensitivity, but high specificity and PPV of TB. Opposite results were recorded for the Vizilite. Although biopsy is the gold standard to detect dysplasia, TB and Vizilite are valuable non-invasive and rapid techniques that aid in clinical detection and screening of dysplasia.

\section{Declaration of Competing Interest}

The Author declares no conflict of interest.

\section{REFERENCES}

1. Brücher M, Björn L and Ijaz S. Transition from normal to cancerous cell by precancerous niche $(\mathrm{PCN})$ induced chronic cell-matrix stress. Oral Oncol. 2019;14: 233-9.

2. Mascitti M, Orsini G, Tosco V and Liu P. An overview on current non-invasive diagnostic devices in oral oncology. Front Physiol. 2018;9: 1510-8.

3. Shukla A, Singh N, Adsul S, Kumar S, Shukla D and Sood A. Comparative efficacy of chemiluminescence and toluidine blue in the detection of potentially malignant and malignant disorders of the oral cavity. J Oral Maxillofac Pathol. 2018;22: 442-7. 
4. Belal M, Elmoneim W, Nasry S, Mostafa B and Ali S. VELscope versus toluidine blue for detection of dysplastic changes in oral keratotic lesions: diagnostic accuracy study. J Arab Soc Med Res. 2018;13: 45-52.

5. Kumari T, Ratna $\mathrm{N}$ and Mujib A. Toluidine blue with a synergistic effect in morphological assessment of oral cytosmears. J Cytology. 2018;35: 185-61.

6. Mehrotra R, Singh M, Thomas S, Nair P, Pandya S, Nigam $\mathrm{N}$, et al. A cross-sectional study evaluating chemiluminescence and autofluorescence in the detection of clinically innocuous precancerous and cancerous oral lesions. J Am Dent Assoc. 2010;41:151-6.

7. Bergholt L, Leth $\mathrm{N}$, Lysdahl H, Lind $\mathrm{M}$ and Foldager C. A standardized method of applying toluidine blue metachromatic staining for assessment of chondrogenesis. Cartilage. 2019;10: 370-4.

8. Patil P, Prakash L and Nikumbh D. Efficacy of toluidine blue staining in cervicovaginal cytology over conventional papanicolaou stain. IP Arch Cyto Histopath Res. 2018;21: 47-51.

9. Liu D, Zhao X, Zeng X, Dan H and Chen Q. Non-invasive techniques for detection and diagnosis of oral potentially malignant disorders. Tohoku J Exp Med. 2016; 238: 165-77.

10. Parakh M, Reddy R and Subramani P. Toluidine blue staining in identification of a biopsy site in potentially malignant lesions: a casecontrol study. Asia Pac J Oncol Nurs. 2017; 4:356-60.

11. Ramanathan A, Deepak T, Manjunath M, Krishna S and Annaji AG. Assessment of oral mucosa in high risk and precancer using chemiluminescent illumination and toluidine blue supravital staining. Int J Oral Health Med Res. 2016;2: 28-32.
12. Parakh M, Reddy J and Subramani P. Toluidine blue staining in identification of a biopsy site in potentially malignant lesions: a case-control study. Asia Pac J Oncol Nurs. 2017;4: 356-60.

13. Adil N, Ali H, Yuwanati M, Singh A, Sawant S and Umarji H. Comparative study on the efficacy of tissue autofluorescence (visually enhanced lesion scope) and toluidine blue as a screening method in oral potentially malignant and malignant lesions. J Med Sci. 2017; 37: 91-8.

14. Güneri $\mathrm{C}$ and Pelin K. Oral leukoplakia and toluidine blue staining. Clin Surg. 2017;4: 1-3.

15. Gogna P, O'Sullivan D and King W. The effect of inflammation-related lifestyle exposures and interactions with gene variants on long interspersed nuclear element-1 DNA methylation. Epigenomics. 2018;10: 785-96.

16. Ahire B, Bhoosreddy A, Bhoosreddy S, Pandharbale A, Kunte V and Shinde M. Awareness of patients about existing oral precancerous lesions/conditions in Nashik city of Maharashtra. J Ind Ass Pub Health Dent. 2016;14: 207-10.

17. Latoo S, Shetty P and Gowhar O. Prevalence of oral precancerous lesions among population of Sasihitlu village, Mukka, Dakshin Kannada district. Int J Cur Res. 2016;08: 2421-3.

18. Gowhar O, Ain T, Singh N and Sultan S. Prevalence of oral premalignant and malignant lesions in moradabad, india - a retrospective study. Int J Cont Med Res. 2016;3: 2079-81.

19. Bagga M, Kumar A and Bhatnagar D. Comparative morphological analysis of precancerous lesions and conditions by clinical examination, chemiluminescence, and toluidine blue. J Indian Acad Oral Med Radiol. 2017;29: 249-53. 